\title{
Effect of gate-driven spin resonance on the conductance of a one-dimensional quantum wire
}

\author{
Almas F. Sadreev ${ }^{1}$ and E. Ya. Sherman ${ }^{2,3}$ \\ ${ }^{1}$ L.V. Kirensky Institute of Physics, 660036, Krasnoyarsk, Russia \\ ${ }^{2}$ Department of Physical Chemistry, Universidad del Pais Vasco UPV-EHU, 48080 Bilbao, Spain \\ ${ }^{3}$ IKERBASQUE, Basque Foundation for Science, Bilbao, Spain
}

(Dated: August 7, 2018)

\begin{abstract}
We consider quasiballistic electron transmission in a one-dimensional quantum wire subject to both time-independent and periodic potentials of a finger gate that results in a coordinate- and time-dependent Rashba-type spin-orbit coupling. A spin dependent conductance is calculated as a function of external constant magnetic field, the electric field frequency, and potential strength. The results demonstrate the effect of the gate-driven electric dipole spin resonance in a transport phenomenon such as spin-flip electron transmission.
\end{abstract}

PACS numbers: 72.20.Dp,72.25.Dc,73.23.Ad

\section{INTRODUCTION}

Since the Datta-Das spin field-effect transistor [1] was proposed, the Rashba spin-orbit interaction (RSOI) [2] has attracted considerable attention on account of its possible applications in spintronics. The manipulation of electron spins can be achieved via an external active control, which is the essential requirement for spintronics devices. Interest in the RSOI as an instrument to electrically manipulate spins in nanosystems [3] has been growing since Nitta et al. [4] showed that in an inverted $\mathrm{In}_{0.53} \mathrm{Ga}_{0.47} \mathrm{As} / \mathrm{In}_{0.52} \mathrm{Al}_{0.48}$ As quantum well the RSOI can be controlled by applying a gate voltage. In general, this control is strongly material and structuredependent, as was demonstrated in more recent experiments on $n$-type semiconductors $5-10]$. A similar effect of electric field has also been achieved in a $p$-type InAs semiconductor, as reported by Matsuyama et al. [11].

Assuming that a finger gate with dc voltage is located above a conducting channel based on a two-dimensional electron gas (2DEG) as shown in Fig. 1 one can see that its electric field gives rise to a local RSOI. Under the assumption of a stepwise RSOI the electron ballistic transport in a quasi one-dimensional wire has undergone a thorough investigation 12 22]. In the present paper we consider an actual electric field produced by the dc biased finger gate that gives rise to a nonuniform RSOI. Next, we assume that the finger gate is also biased by time-dependent (ac) voltage that affects the space and time-dependent RSOI. Electron transport through wires with spin-orbit interaction subjected to a time-periodic potential was studied in Refs. 23, 24].

An ac biased finger gate contributes to the timeperiodic RSOI which may give rise to many interesting effects such as generation of spin current [25 27] and spinpolarized wavepackets [28]. A well-known and particularly powerful way of manipulating spins in doped III-V heterostructures is electric dipole induced spin resonance (EDSR) [29 33] or gate-driven resonance [34] where the fields coherently driving the spins are electric rather than

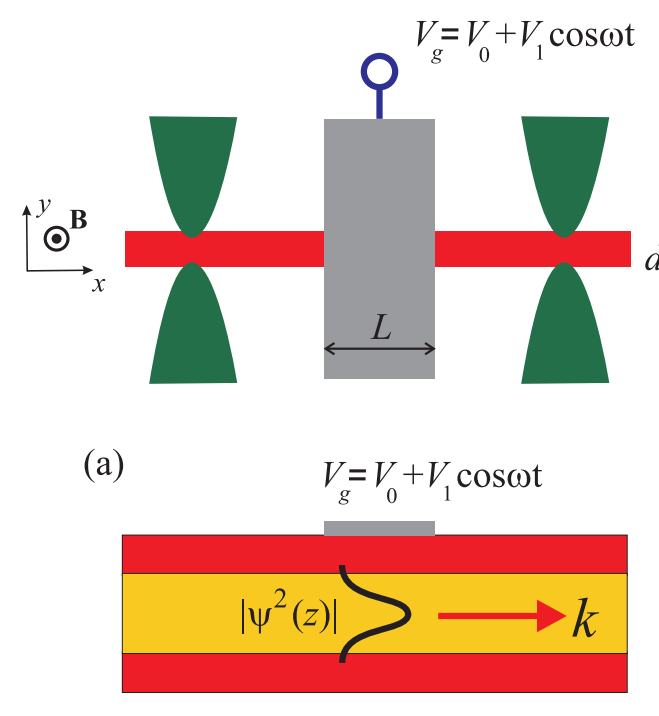

(b)

FIG. 1: (a) View of the conducting channel subject to a dc potential of the quantum point contact gates and dc and ac potential of a finger gate. The inhomogeneous electric field created by the finger gate (shown in gray) represents the scattering active region. The distance between pairs of quantum point contacts, $L_{w}$, is not shown here. (b) Crosssection of the nanostructure. The internal layer shows the propagation channel, $k$ is the electron momentum, and $\psi(z)$ is the wave function of the localized electron. The gates for $y$ - axis confinement are not presented. The details of the figure are not to scale.

magnetic such as in a conventional paramagnetic resonance. Nowack et al. [35] observed EDSR in a single GaAs quantum dot and found that, as expected, the Rabi frequency for spin flips is much less than the corresponding Zeeman splitting. Kato et al. 36] manipulated electron spins in a parabolic AlGaAs quantum well by a $\mathrm{GHz}$ bias applied to a single gate producing a field $E(t)$ perpendicular to the well. Pioro-Ladriere et al. [37] studied the effect of a slanting magnetic field for the EDSR. 
All these effects were observed for electrons localized in quantum dots. Although it is clear that the electron spatial dynamics, e.g., in a double quantum dot can strongly modify the Rabi frequency of the EDSR [38, 39], there is no understanding of the signatures of the EDSR in the electron transport. Here we study ballistic electron transport in a one-dimensional (1D) quantum wire subject to dc and ac bias produced by a finger gate and a Zeeman magnetic field and demonstrate the role of the EDSR in the conductance of such a system. We found that the effect leads to avoiding crossing in the dependence of the conductance on the electron energy and ac field frequency, or equivalently, the Zeeman splitting.

\section{DESCRIPTION OF MODEL}

The semiconductor structure of our interest is shown in Fig. 11which demonstrates that in the absence of a gate bias the system is symmetric with respect to the $z \rightarrow-z$ reflection. Then the system has only the Dresselhaus SOI because of the host crystal electric field [40, 41], $H_{D}=$ $i \beta\left\langle k_{z}^{2}\right\rangle\left[\sigma_{x} \partial_{x}-\sigma_{y} \partial_{y}\right]$, over the whole sample. Next, similar to Ref. 42], we consider a metallic gate at a height $h$ from the conducting channel whose length $L_{w}$ along the transport $x$-axis exceeds the gate width $L$, such that their ratio $l \equiv L_{w} / L \geq 1$. The electrostatic potential of the biased metallic gate was derived by Davies et al. [43]. For the structure presented in Fig. 1, the gate potential has the form

$$
\begin{array}{r}
V(x, z, t)=\left(V_{0}+V_{1} \cos \omega t\right) \phi(x, z), \\
\phi(x, z)=\frac{1}{\pi}\left[\arctan \frac{L+x}{z}+\arctan \frac{L-x}{z}\right],
\end{array}
$$

where the gate is chosen as the origin of $z$. The gate potential produces the RSOI nonuniform over $x$, [4, 45]

$$
H_{R}=-i \alpha\left[\mathbf{E}(\boldsymbol{\sigma} \times \boldsymbol{\nabla})-\frac{1}{2} \boldsymbol{\sigma}(\boldsymbol{\nabla} \times \mathbf{E})\right],
$$

where $\mathbf{E}(x, y, z, t)=-\nabla V(x, z, t)-\nabla V_{\mathrm{LC}}(y)$. Here $V_{\mathrm{LC}}(y)$ is the lateral confining potential [46], and the last contribution in Eq. (2) ensures the hermiticity of the RSOI. The $y$-axis confinement length $d$ is typically tens to hundreds of nanometers. This small length establishes a corresponding high energy gap for the transverse excitations and protects the system from exciting the transverse modes. In what follows we adopt strong lateral confinement of about tens of nanometers in order to focus on the effects of the ac potential for the $x$-axis electron transmission through the one-dimensional quantum wire. Then we can restrict ourselves to the ground state $\psi_{0}(y, z)$ which is a sharp function compared to the characteristic scale along the wire $L_{w}$ which is taken to be of the order of hundreds of nanometers.

The projection of the total Hamiltonian onto that ground state gives us the following effectively one- dimensional Hamiltonian

$$
\begin{aligned}
\widetilde{H} & =\int d y d z \psi_{0}(y, z) H \psi_{0}(y, z) \\
& =\varepsilon_{0}\left[\widetilde{H}_{0}+\widetilde{V}_{0}(x, t)+\widetilde{H}_{Z}+\widetilde{H}_{R}\right] .
\end{aligned}
$$

Here

$$
\begin{aligned}
& \widetilde{H}_{0}=-\frac{\partial^{2}}{\partial x^{2}}, \\
& \widetilde{V}_{0}(x, t)=\left(v_{0}+v_{1} \cos \omega t\right) \phi(x, z=h)
\end{aligned}
$$

are the dimensionless Hamiltonian of free motion of electrons and the dimensionless potential of the finger gate, respectively. The coordinate $x$ is measured in terms of the gate width $L$ and the energy is measured in units of $\varepsilon_{0}=\hbar^{2} / 2 m^{*} L^{2}$. The Zeeman contribution is

$$
\widetilde{H}_{Z}=B \sigma_{z}
$$

where $B=g \mu_{B} H_{\text {ext }} / 2 \varepsilon_{0}$ is the dimensionless magnetic field applied perpendicular to the wire as shown in Fig. 1 (a). Here $g$ is the $g$-factor, and $H_{\text {ext }}$ is the magnetic field. We assume that the magnetic length is much larger than the channel width $d$ and neglect the influence of the magnetic field on the orbital motion.

The term

$$
\widetilde{H}_{R}=i \varepsilon_{0} \sigma_{y}\left[\widetilde{\alpha}(x, t) \frac{\partial}{\partial x}+\frac{1}{2} \frac{\partial \widetilde{\alpha}(x, t)}{\partial x}\right]
$$

constitutes the time-periodic RSOI where

$$
\begin{aligned}
& \widetilde{\alpha}(x, t)=\widetilde{\alpha}\left(v_{0}+v_{1} \cos \omega t\right) e(x), \\
& e(x)=\frac{1}{\pi}\left[\frac{1+x}{h^{2}+(1+x)^{2}}+\frac{1-x}{h^{2}+(1-x)^{2}}\right],
\end{aligned}
$$

as follows from the dc and ac gate potentials (1). We use dimensionless variables with $\widetilde{\alpha}=(1 \mathrm{~V} \times \alpha) / \varepsilon_{0} L^{2}$, and for practical purposes take $1 \mathrm{~V} / L$ as the unit of the electric field. All distances are measured in terms of $L$, the magnetic field is measured in terms of $2 \varepsilon_{0} / g \mu_{B}$ and the frequency $\omega$ is measured in terms of $\varepsilon_{0} / \hbar$, respectively. We assume $\hbar \equiv 1$ below in the text, if not explicitely stated otherwise. Profiles of the dimensionless electric field $e(x)$ are plotted in Fig. 2 for different distances $h$ of the finger gate from the channel, which shows that the RSOI mainly contributes at the edges of the finger gate for small height $h$. We employ here the representation:

$$
\sigma_{x}=\left(\begin{array}{cc}
0 & -i \\
-i & 0
\end{array}\right), \quad \sigma_{y}=\left(\begin{array}{cc}
1 & 0 \\
0 & -1
\end{array}\right), \quad \sigma_{z}=\left(\begin{array}{cc}
0 & 1 \\
1 & 0
\end{array}\right)
$$

The anticommutator form $(i / 2)\{\widetilde{\alpha}(x, t), \partial / \partial x\}$ in Eq. (7), which is related to the inhomogeneous Rashba field $\widetilde{\alpha}(x, t)$ is often adopted by a phenomenological application of the Dirac symmetrization rule for a product of noncommuting operators or it is taken for granted [16, 47]. In addition, one can see that the RSOI caused by 

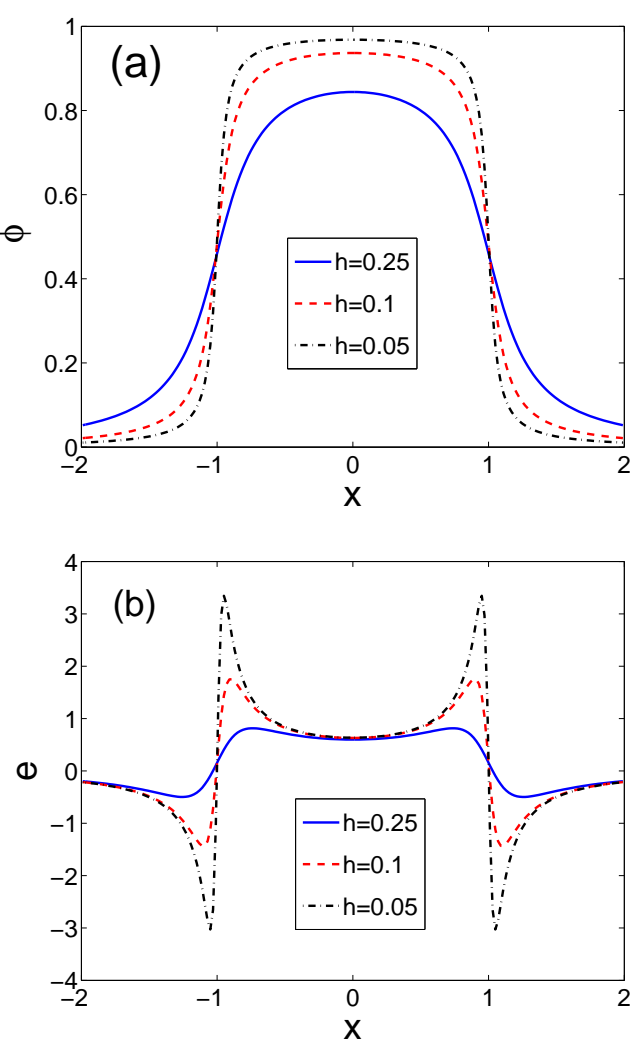

FIG. 2: Profiles of the dc potential $\phi(x)$ and electric field $e(x)$ which define the non-uniform Rashba SOI in Eq. (8) over the transport axis for different distances between the finger gate and the 2DEG. The potential is measured in terms of $\varepsilon_{0}$ given here.

the lateral confinement is excluded because the electric field at the position $y=0$ of the thin wire vanishes.

The values of the quantities necessary to describe the transport are collected in Table I. To be specific, we consider typical Rashba and Dresselhaus SOI constants, effective masses, and $g$-factors for the InAs- and InSbbased heterostructures [45, 48]. As seen from the table, the Dresselhaus SOI can be neglected in these semiconductor structures even at rather weak applied fields. In addition, we present the characteristic Zeeman field and frequency corresponding to $\varepsilon_{0}$ for the finger gate length $L=1000 \AA$.

We begin with a stationary transmission for $v_{1}=0$ and $B=0$ and assume solely for this example that the gate covers the entire channel, that is, $l=1$. In this geometry we achieve the resonance transmission when the electron energy matches the corresponding eigenenergy of the gated wire channel. We assume that the Rashba coupling is homogeneous over the wire subjected to the homogeneous potential $v_{0}$ according to Eq. (11). As a result, the dependence of the resonances on the applied potential becomes, in general, parabolic due to the linear contribution of the Coulomb field and quadratic in $v_{0}$ contribution of the spin-orbit coupling.
We show the resonance peaks of the transmission which follow the eigenenergies of the 1D Rashba box by dashed lines in Fig. 3. As expected, for $\widetilde{\alpha}=0$ the resonant transmission demonstrates linear behavior with $v_{0}$ [Fig. [3(a)]. For $\widetilde{\alpha} \neq 0$ the behavior of the eigenenergies of a closed wire with $v_{0}$ is parabolic [Fig $\left.3(\mathrm{~b})\right]$. Respectively, the resonance behavior of the Rashba wire demonstrates similar behavior as shown in Fig. 3 (b) for $\widetilde{\alpha}=0.75$. In full agreement with the rigorous results in Refs. [49, 50] the numerically calculated spin polarization

$$
P=\frac{G_{\uparrow \uparrow}+G_{\uparrow \downarrow}-G_{\downarrow \uparrow}-G_{\downarrow \downarrow}}{G_{\uparrow \uparrow}+G_{\uparrow \downarrow}+G_{\downarrow \uparrow}+G_{\downarrow \downarrow}}
$$

vanishes because of the single-channel transmission in the $1 \mathrm{D}$ wire.
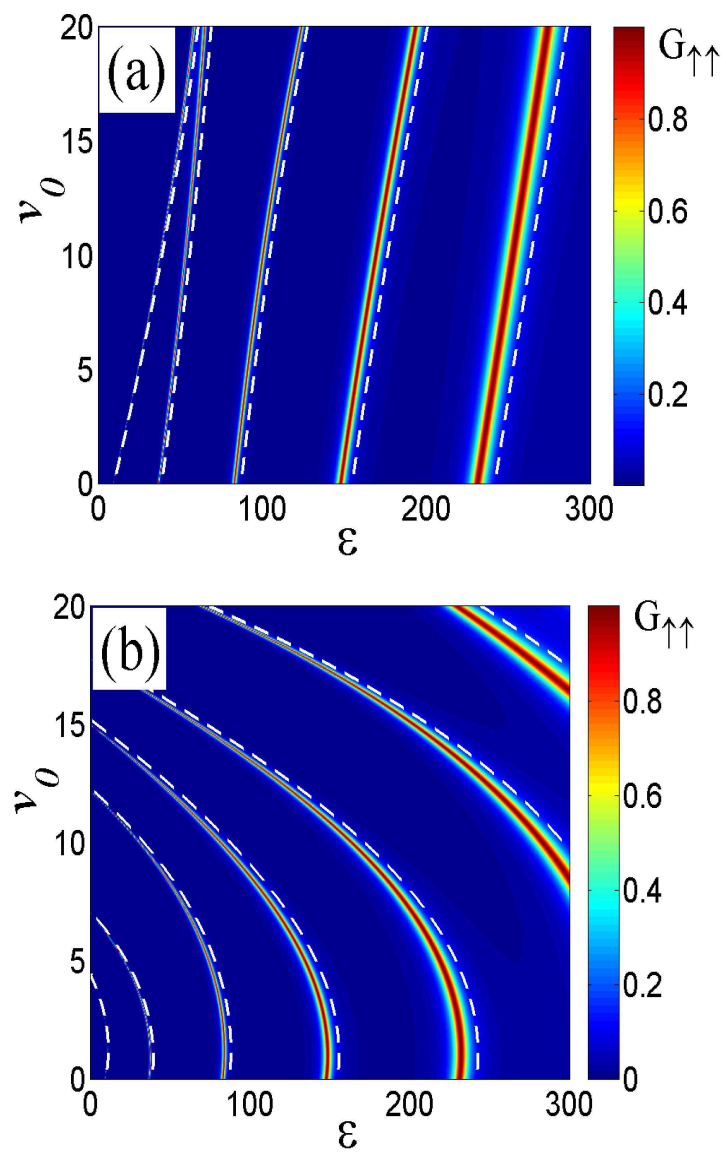

FIG. 3: Conductance $G_{\uparrow \uparrow}$ of the 1D wire vs incident energy $E$ and dc potential applied to finger gate (11) $v_{0}$ for $v_{1}=0, h=$ $0.1, l=1$ (a) $\widetilde{\alpha}=0$ and (b) $\widetilde{\alpha}=0.75$. The incident energy and gate potential are measured in terms of $\varepsilon_{0}$. Eigenenergies of closed 1D Rashba wire for these parameters are shown by dashed white lines. 
TABLE I: Parameter sets of the InAs- and InSb based heterostructures for the gate length $L=1000 \AA$.

\begin{tabular}{|c|c|c|c|c|c|c|c|c|c|}
\hline Structure & $m^{*}\left[m_{0}\right]$ & $\alpha\left[e \AA^{2}\right]$ & $\beta\left[\mathrm{eV} \cdot \AA^{3}\right]$ & $\varepsilon_{0}[\mathrm{meV}]$ & $\widetilde{\alpha}$ & $\beta$ & $g$ & $B\left[\right.$ for $\left.H_{\text {ext }}=1 \mathrm{~T}\right]$ & $\omega / 2 \pi[\mathrm{GHz}]$ \\
\hline InAs & 0.023 & 117 & 27 & 0.15 & 0.7 & $1.8 \times 10^{-4}$ & 8 & 1.55 & 36 \\
\hline InSb & 0.035 & 523 & 760 & 0.23 & 3 & $3.3 \times 10^{-3}$ & -10 & 1.26 & 55 \\
\hline
\end{tabular}

\section{AC ASSISTED SPIN-DEPENDENT ELECTRON TRANSMISSION}

Here we consider the spin-dependent transmission of electrons through the 1D wire subjected to a dc and ac potential of the finger gate (1). Before providing a detailed numerical analysis, we address qualitatively an important question regarding whether a spin polarization can appear in this situation in the absence of an external magnetic field. For stationary single-channel transmission in the quantum wire the RSOI cannot give rise to spin polarization [49, 50]. However, the ac timeperiodic RSOI (8) opens additional spin dependent channels of electron transmission at the Floquet quasienergies $\varepsilon+m \omega, m=0, \pm 1, \ldots$ Numerical calculations in Refs. [26, 47] show the spin polarization for the case of the stepwise time-periodic RSOI. We argue that there is no spin polarization for smooth coordinate behavior of the timeperiodic RSOI, at least, for zero Dresselhaus SOI. Indeed, for that case the only spin component in the RSOI is $\sigma_{y}=\sigma$, which is an integral of motion. Then as Eq. (3) shows the electron transmission with spin $\sigma=1(\uparrow)$ is not mixed with the transmission with $\sigma=-1(\downarrow)$. From Eq. (3) it follows that the electron transmissions with spin $\sigma= \pm 1$ differ by only half of the time period $\pi / \omega$. And therefore, after a time average of the conductances, $G_{\sigma \sigma}$ do not depend on $\sigma$ while there is no conductance $G_{\sigma,-\sigma}$ with spin flip. Thus this simple consideration proves that the spin polarization (11) equals zero. Below we show that numerical computations agree with that consideration for smooth space behavior of the time-periodic RSOI.

The procedure of calculating the electron transmission through the time-periodic potential (photon-assisted transmission) is well described in literature [51 56].
There are two time-dependent contributions in our system. The first one is the periodic oscillations of the potential produced by the ac biased finger. This effect was considered in many publications for a spatially stepwise time-periodic potential [51 53, 55]. The second one is the oscillating RSOI, considered for the stepwise spatial dependence of the ac finger field in Refs. [26, 47].

We use the tight-binding approximation to calculate the conductance through the space and time dependent profiles of the potential [56 59]. In the leads where there is no SOI the wave functions can be written as [55, 6062 ,

left:

$\psi_{j \sigma}(t)=\sum_{m \sigma^{\prime}} \frac{e^{-i(\varepsilon+m \omega) t}}{\sqrt{2 \pi \rho\left(k_{m}\right)}}\left[\delta_{m, 0} \delta_{\sigma \sigma^{\prime}} e^{i k_{0} j}+r_{m \sigma \sigma^{\prime}} e^{-i k_{m} j}\right]$,

right:

$\psi_{j \sigma}(t)=\sum_{m \sigma^{\prime}} \frac{e^{-i(\varepsilon+m \omega) t}}{\sqrt{2 \pi \rho\left(k_{m}\right)}} t_{m \sigma \sigma^{\prime}} e^{i k_{m} j}$

where

$$
\varepsilon+m \omega=-2 \cos k_{m}, \rho\left(k_{m}\right)=\partial \varepsilon / \partial k_{m}
$$

Here we imply that the electron enters from the left lead with energy $\varepsilon$ and spin $\sigma$ and reflects and transmits with energy $\varepsilon+m \omega$ and spin state $\sigma^{\prime}$ with corresponding reflection and transmission amplitudes $r_{m, \sigma \sigma^{\prime}}$ and $t_{m, \sigma \sigma^{\prime}}$, respectively. We assume that the Zeeman and Rashba fields affect the conducting electron in the $1 \mathrm{D}$ wire only. Inside the $1 \mathrm{D}$ wire of length $L=a_{0} N$ with coordinate $x_{j}=a_{0} j, j=1,2, \ldots, N$, we present the Schrödinger equation according to Eqs. (3)-(8) as follows:

$$
\begin{aligned}
& (\varepsilon+m \omega) \psi_{m, j \sigma}+\frac{\psi_{m, j+1 \sigma}+\psi_{m, j-1 \sigma}-2 \psi_{m, j \sigma}}{a_{0}^{2}}-v_{0} \phi_{j} \psi_{j, m \sigma}-v_{1} \phi_{j}\left(\psi_{j, m+1 \sigma}+\psi_{j, m-1 \sigma}\right)-B \psi_{m, j,-\sigma} \\
& -i \widetilde{\alpha} \sigma v_{0} e_{j} \frac{\psi_{m, j+1 \sigma}-\psi_{m, j-1 \sigma}}{2 a_{0}}-i \widetilde{\alpha} \sigma v_{1} e_{j} \frac{\psi_{m+1, j+1 \sigma}+\psi_{m-1, j+1 \sigma}-\psi_{m+1, j-1 \sigma}-\psi_{m-1, j-1 \sigma}}{2 a_{0}}=0 .
\end{aligned}
$$

Here $\phi_{j} \equiv \phi\left(x_{j}, h\right)$ and $e_{j} \equiv e\left(x_{j}\right)$. To simulate entering and exiting through quantum point contacts we implied the hopping matrix element $t=0.5$ between the leads and the 1D. In what follows we take the ratio $l=4.0$.

We define the transmission (reflection) probability as the ratio of output current at the right lead to the input current, where the current is defined in conventional units as

$$
J_{j \sigma \sigma^{\prime}}=J_{0} \overline{\operatorname{Im}\left[\psi_{j \sigma}^{*} \psi_{j+1, \sigma^{\prime}}\right]}, \quad J_{0}=\frac{e \hbar}{2 m^{*} L} .
$$


Here $\cdots \equiv(\omega / 2 \pi) \int_{0}^{2 \pi / \omega} \cdots d t$. Substituting Eqs. (12) into Eq. (15) we obtain the dimensionless conductance

$$
G_{\sigma \sigma^{\prime}}=\sum_{m} \frac{\sin \operatorname{Re}\left(k_{m}\right)\left|t_{m \sigma \sigma^{\prime}}\right|^{2}}{\sin k_{0}} .
$$

That expression reduces to the standard expression for the conductance in the continuum approximation [55]. Taking the real part of $k_{m}$ in the nominator of Eq. (16) assures that the Floquet states with quasienergies $\varepsilon+m \omega$ beyond the propagation band having an imaginary wave vector $k_{m}$ cannot participate in the conductance.

\section{NUMERICAL RESULTS}

In our numerical computations we chose the numerical lattice unit $a_{0}=0.01$. For the dimensionless energies of electron $\varepsilon \sim 100$ the characteristic wavelength is of the order 1 , which greatly exceeds $a_{0}$. The next condition for $a_{0}$ is that $a_{0} \ll \Delta x$ where $\Delta x$ is a characteristic scale over which the potential and electric field of the finger gate undergo sharp changes as shown in Fig. 2 This scale is close to the distance $h$ between the gate and the channel. Therefore the condition for the numerical lattice unit is $a_{0} \ll h$, which is satisfied also if we take $h=0.1$. There is also the condition for the dimensionless frequency $\omega>v_{1} / M$ where $2 M+1$ is the number of Floquet states [54, 59]. In numerics we consider only the minimal case $M=1$ to reduce the time of the numerical calculations which means the region of small frequencies $\omega<v_{1}$ is excluded from consideration.

\section{A. Conductance in AC and DC biased wire}

Figure 4 shows the electron conductance of quantum wire subjected to a finger gate that is dc and ac biased (Fig. 1) for the incident energies substantially exceeding the effective potential barrier height. Switching off the ac potential, $v_{1}=0$, one obtains the resonance transmission for $\varepsilon \approx \varepsilon_{n}$ where $\varepsilon_{n}$ are the eigenenergies of the closed wire with an applied dc potential. These eigenenergies are marked in Fig. 4 by solid red circles. The resonance positions, however, are slightly shifted because of the openness of the 1D wire. Then the application of the ac potential gives rise to the quasi energies $\varepsilon_{n} \pm \sqrt{2 v_{1}^{2}+\omega^{2}}[59]$. The coincidence in these quasienergies with the basic eigenenergies $\varepsilon_{n^{\prime}}$ results in avoiding crossing as seen from Fig. 4 (a). Note because of the even symmetry of the potential $\phi(x, z)$ relative to an inversion of the $x$-axis the only Floquet states that avoid the basic energies are the ones which have the same parity, i.e., $n^{\prime}=n \pm 2, n \pm 4, \ldots$ as seen from Fig. 4(a).

Switching on the RSOI induced by the dc and ac electric fields gives rise to the new selection rules of the avoiding crossing of the Floquet states. The time-periodic term $v_{1}\{\widetilde{\alpha}(x), k\} / 2 \times \cos \omega t$ in the Rashba Hamiltonian
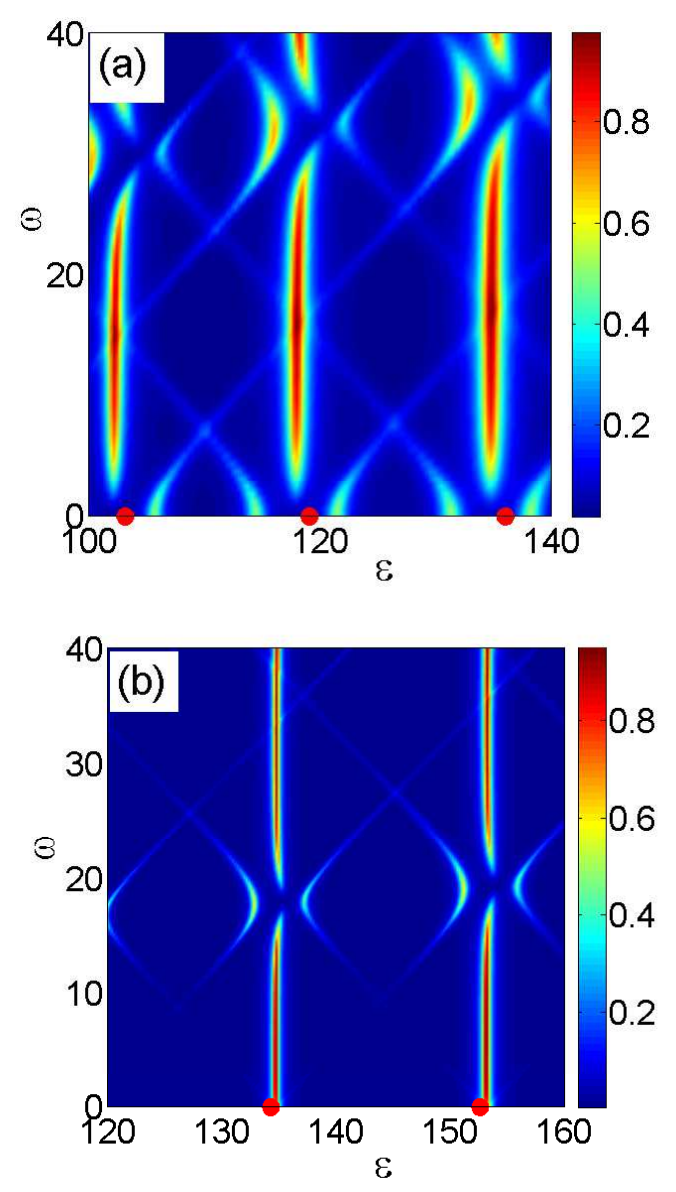

FIG. 4: Conductance $G_{\uparrow \uparrow}$ vs incident energy and frequency of the ac potential for one gate with the parameters $v_{0}=1, v_{1}=$ $0.25, l=4$. (a) $\widetilde{\alpha}=0$ and (b) $\widetilde{\alpha}=1$. The solid red circles mark the eigenenergies of the closed $1 \mathrm{D}$ wire.

(77) is odd with respect to the $x$-inversion. Therefore it mixes the neighboring eigenstates with the opposite parity of the closed wire while the time-periodic potential $v_{1} \phi(x) \cos \omega t$ term mixes the eigenstates with the same parity. As a result we obtain the avoiding crossings of nearest neighbor resonances shown in Fig. 4 (b). With the growth of the potential amplitude $v_{0}$ the avoiding crossings occur irrespective of the selection rules that give rise to more complicated frequency behaviors of the conductance.

It is possible to exclude the time-periodic perturbation of the potential by applying two oppositely biased finger gates symmetrically disposed up and below the conducting layer. Then the electron experiences only the timeperiodic RSOI without a coordinate-dependent potential energy. For a given ac finger bias, here the effects of avoiding crossings are expected to be stronger due to the doubling of the electric field affecting the RSOI. 


\section{B. AC affected spin resonance for transmission in magnetic field}

Now we apply the magnetic field perpendicular to the quantum wire as shown in Fig. 1 (a). For the dc potential the term (6) obviously gives rise to the Zeeman splitting of the energy levels of the wire. Respectively, the resonance transmission follows these split energy levels as shown in Fig. 5. For $\alpha=0$ the conductance simply follows the magnetic field as seen from Fig. 5 (a), while the RSOI leads to avoiding crossing behavior of the conductance because of $\left[\widetilde{H}_{R}, \widetilde{H}_{Z}\right] \neq 0$, as seen from Figs. 5 (b), 5 (c), and 5 (d).

The most important point is that the last term in the Hamiltonian (3) has similar effects as the radio frequency magnetic field directed perpendicular to the constant Zeeman field. Therefore we can expect signatures of spin resonance for $\omega \approx 2 B$ with spin inversion 2931 . We take $B=5$, which is shown by the dashed line in Fig. 5] (c). Figure 6] (a) shows the conductance $G_{\uparrow \downarrow}$ vs energy and frequency of the ac potential with the RSOI $\widetilde{\alpha}=1$. For $\widetilde{\alpha}=0$ this conductance is zero and therefore is not presented. One can see that the basic resonances in conductance follow the RSOI and Zeeman split eigenenergies, shown in Fig. 5 (c) as open circles. However, there is a fine structure of the conductance in the form of avoiding crossings where the Floquet resonances cross the basic resonances, which are marked in Fig. 5 (c) by the open circles. That indicates spin resonances for swiping the frequency of the ac potential. Figure 6 (b) shows the conductance $G_{\uparrow \downarrow}$ for the fixed frequency of the ac potential $\omega=10$ vs incident energy and a constant magnetic field applied perpendicular to the transport axis $x$. Similar to the case in Fig. 6 (a) we see the self-avoiding Floquet resonances with the basic Zeeman peaks of the conductance shown in Fig. 5] (d). This result clearly shows spin resonances affected by the electric ac potential.

An interesting feature of the transmission in a nonzero magnetic field, where the time-reversal symmetry is broken, is the difference between two spin-flip channels, that is $G_{\uparrow \downarrow}-G_{\downarrow \uparrow}$. It is presented in Fig. 7 and corresponds to $G_{\uparrow \downarrow}$ in Fig. 6. Although this difference is small, being of the order of 0.1 of $G_{\uparrow \downarrow}$, and appears mainly in the anticrossing spin-flip domains of Fig. 6, its nonzero value is the qualitative manifestation of the broken time-reversal symmetry, and, as a result, of the possible generation of finite spin polarization [see Eq.(11)].

\section{SUMMARY AND DISCUSSION}

We studied the effects of dc and ac biased finger gate on the resonant transmission of an electron through a 1D quantum wire. The potential and the electric field of the gate are local as shown in Fig. 2. The ac field of the gate forms a time-periodic Rashba SOI which can cause spin flip for the electron that is transmitted through the
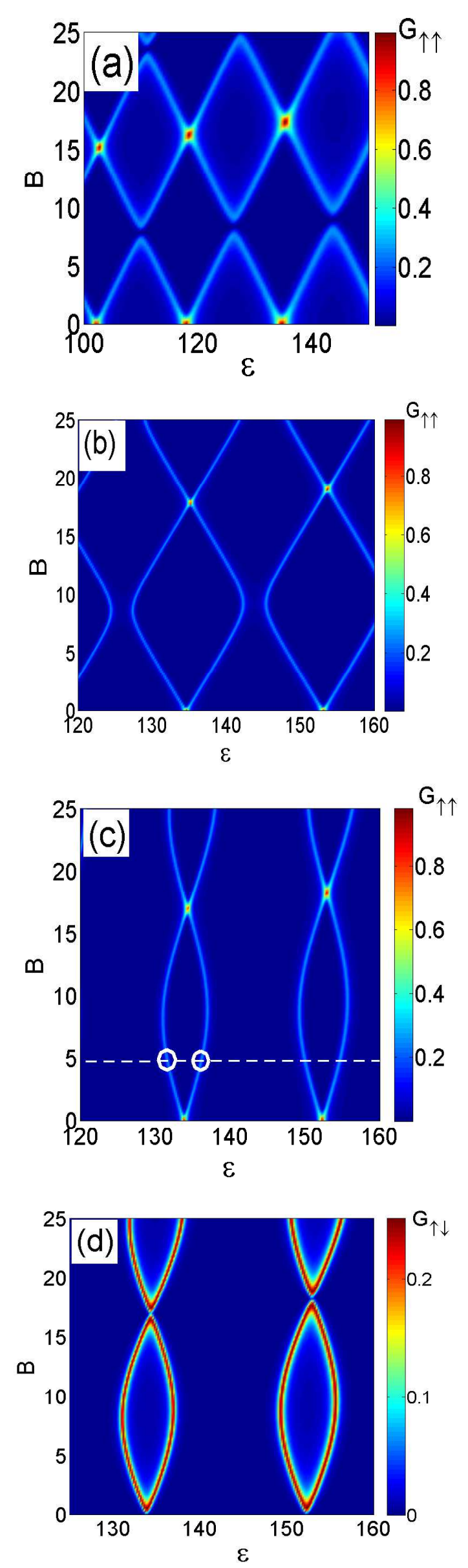

FIG. 5: The stationary spin-dependent conductances $G_{\sigma \sigma^{\prime}}$ vs magnetic field and energy for $\widetilde{\alpha}=0$ (a), $\widetilde{\alpha}=0.25$ (b), $\widetilde{\alpha}=1$ (c) and (d). $v_{0}=1$. 

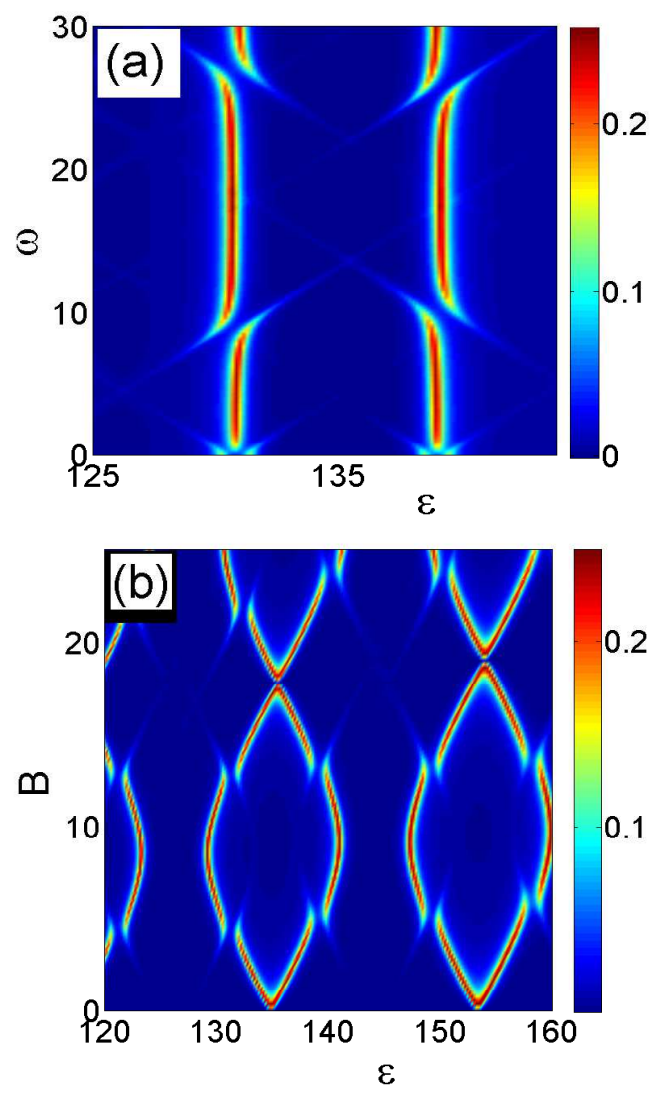

FIG. 6: The ac affected conductance $G_{\uparrow \downarrow}$ (a) vs incident energy and frequency of the ac potential when the magnetic field $B=5$ is applied perpendicular to the wire and (b) vs incident energy and external magnetic field for $\omega=10$. The parameters are $v_{0}=1, v_{1}=0.25, l=4, \widetilde{\alpha}=1$.

gated channel. This results in new features in the spinflip electron conductance $G_{\uparrow \downarrow}$ such as the Floquet satellites and self-avoiding crossing of resonances, while the basic resonances follow the eigenenergies of a closed 1D wire subject to a dc potential, a Zeeman magnetic field applied across the wire, and a static RSOI. The simplest resonance-induced transition corresponds to the matching of the frequency-dependent Floquet resonance peak with the basic resonance peak corresponding to the Zeeman splitting. That results in a spin resonance that is similar to that formulated by Rashba and Efros when the time-periodic electric field gives rise to a spin flip in a constant magnetic field with spatially uniform spin-orbit coupling [31]. As can be seen in Fig. 6, the characteristic static dimensionless magnetic field and gate frequency in our model consideration are of the order of 10. According to Table \ these numbers correspond to ten Tesla and a few hundreds $\mathrm{GHz}$ (in the upper range of the microwave radiation), respectively. In addition, the characteristic required electric fields are of the order of $10^{4} \mathrm{~V} / \mathrm{cm}$. In practice, these parameters are strongly system-dependent and the effect, studied here only semiquantitatively, can be possibly observed at lower fields
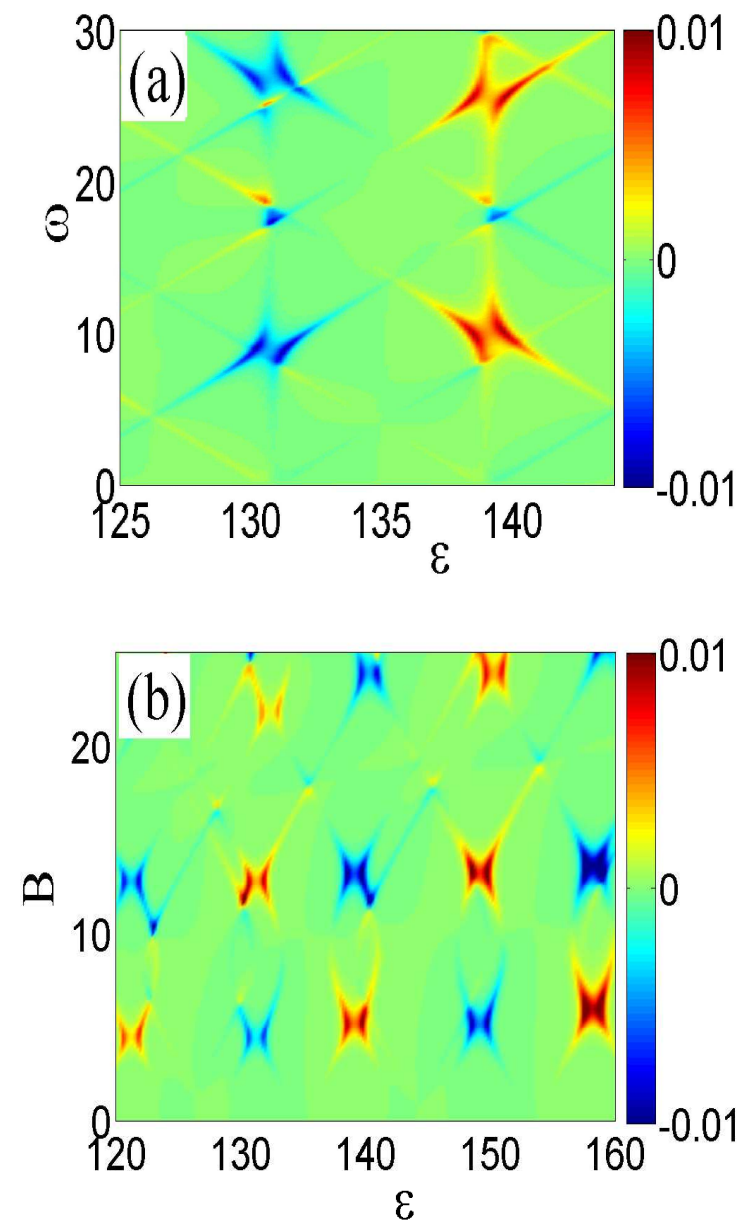

FIG. 7: The difference $G_{\uparrow \downarrow}-G_{\downarrow \uparrow}$ vs incident energy and frequency of the ac potential when the magnetic field $B=5$ is applied perpendicular to the wire and (b) vs incident energy and an external magnetic field for $\omega=10$.

and frequencies.

As additional conclusions, we would like to comment on the possibility of producing spin polarization in electron single channel transmission by the ac field. As it was argued in Sec. III the time-periodic Rashba SOI cannot lead to spin polarization for the transmission through a $1 D$ wire at $B=0$. This result agrees with our computer simulations but disagrees with the numerical results of Ref. 47] where spin polarization around 0.2 was found for zero Dresselhaus SOI $\beta=0$. An origin of the difference is related to the coordinate dependence of the finger gate field. Numerical calculations show a tendency for decreasing the spin polarization with decreasing the simulation lattice constant $a_{0}$ and increasing the height $h$, when the electric field and potential become smooth. At $a_{0} \ll h$ the spin polarization becomes negligibly small. Thus the stepwise approximation of such a non uniform RSOI conceals a danger for numerical com- 
putations based on finite difference schemes.

The manifestation of the electric dipole spin resonance in the ballistic transport through a one-dimensional channel can help in the design of devices with a spin transport controlled by an electric field in quantum nanoscale and mesoscopic systems.

\section{Acknowledgments}

We have benefited from discussions with E.A. de Andrada e Silva and Roland Winkler. The work of AS was partially supported by the RFBR grant 13-02-00497. EYS acknowledges support from the University of Basque Country UPV/EHU under program UFI 11/55, Spanish MEC (FIS2012-36673-C03-01), and "Grupos Consolidados UPV/EHU del Gobierno Vasco" (IT-472-10).
[1] S.Datta and B.Das, Appl. Phys. Lett. 56, 665 (1990).

[2] E.I. Rashba, Sov.Phys. Solid State 2, 1109 (1960); Y.A. Bychkov and E.I. Rashba, J.Phys. C 17, 6039 (1984).

[3] H.C. Koo, J.H. Kwon, J. Eom, J. Chang, S.H. Han, and M. Johnson, Science, 325, 1515 (2009).

[4] J. Nitta, T. Akazaki, H. Takayanagai, and T. Enoki, Phys. Rev. Lett. 78, 1335 (1997).

[5] J.P. Heida, B.J. van Wees, J.J. Kuipers, T.M. Klapwijk, and G. Borghs, Phys. Rev. B 57, 11911 (1998).

[6] G. Engels, J. Lange, Th. Schäpers, and H. Lüth, Phys. Rev. B 55, 1958 (1997).

[7] Th. Schäpers, G. Engels, J. Lange, Th. Klocke, M. Hollfelder, and H. Lüth, J. Appl. Phys. 83, 4324 (1998).

[8] C.-M. Hu, J. Nitta, T. Akazaki, H. Takayanagai, J. Osaka, P. Pfeffer, and W. Zawadski, Phys. Rev. B 60, 7736 (1999).

[9] D. Grundler, Phys. Rev. Lett. 84, 6074 (2000).

[10] P. S. Eldridge, W. J. H. Leyland, P. G. Lagoudakis, O. Z. Karimov, M. Henini, D. Taylor, R. T. Phillips, and R. T. Harley, Phys. Rev. B 77, 125344 (2008).

[11] T. Matsuyama, R. Kürsten, C. Messner, and U. Merkt, Phys.Rev. B 61, 15588 (2000).

[12] Y. V. Pershin, J. A. Nesteroff, and V. Privman, Phys. Rev. B 69, 121306 (2004).

[13] X.F. Wang, Phys. Rev. B 69, 035302 (2004).

[14] L. Zhang, P. Brusheim, and H.Q. Xu, Phys. Rev. B 72, 045347 (2005).

[15] L. Serra, D. Sánchez, and R. Lopez, Phys. Rev. B 72, 235309 (2005).

[16] D. Sánchez and L. Serra, Phys. Rev. B 74, 153313 (2006).

[17] S.J. Gong and Z.Q. Yang, J. Appl. Phys. 102, 033706 (2007).

[18] D. Sánchez, L. Serra, and M.-S. Choi, Phys. Rev. B 77, 035315 (2008).

[19] B. Srisongmuang, P.Pairor, and M. Berciu, Phys. Rev. B 78, 155317 (2008).

[20] L. Chirolli, D. Venturelli, F. Taddei, R. Fazio, and V. Giovannetti, Phys. Rev. B 85, 155317 (2012).

[21] G. Thorgilsson, J.C. Egues, D. Loss, and S.I. Erlingsson, Phys. Rev. B 85, 045306 (2012).

[22] Yue Ban and E.Ya. Sherman, J. Appl. Phys. 113, 043716 (2013).

[23] C.S. Tang, Y.H. Tan, and C.S. Chu, Phys. Rev. B 67, 205324 (2003).

[24] B.H. Wu and J.C. Cao, Phys. Rev. B 73, 245412 (2006).

[25] A.G. Malshukov, C.S. Tang, C.S. Chu, and K.A. Chao,
Phys. Rev. B 68, 233307 (2003).

[26] L.Y. Wang, C.S. Tang, and C.S. Chu, Phys. Rev. B 73, 085304 (2006).

[27] C.S. Tang, Int. J. Mod. Phys. B 20, 869 (2006).

[28] V. Szaszko-Bogar, P. Foldi, and F.M. Peeters, arXiv:1308.2552

[29] E.I. Rashba and Al.L. Efros, Phys. Rev. Lett. 91, 126405 (2003).

[30] E.I. Rashba, J. Supercond. 18, 137 (2005).

[31] Al.L. Efros and E.I. Rashba, Phys. Rev. B 73, 165325 (2006).

[32] V.N. Golovach, M. Borhani, and D. Loss, Phys. Rev. B 74, 165319 (2006).

[33] Rui Li, J. Q. You, C. P. Sun, and Franco Nori, Phys. Rev. Lett. 111, 086805 (2013).

[34] E.A. Laird, C. Barthel, E.I. Rashba, C.M. Marcus, M.P. Hanson, and A.C. Gossard, Phys. Rev. Lett. 99, 246601 (2007).

[35] K.C. Nowack, F.H.L. Koppens, Yu.V. Nazarov, and L.M.K. Vandersypen, Science 318, 1430 (2007).

[36] Y. Kato, R.C. Myers, A.C. Gossard and D.D. Awschalom, Nature 427, 50 (2004).

[37] M. Pioro-Ladriere, T. Obata, Y. Tokura, Y.-S. Shin, T.Kubo, K. Yoshida, T. Taniyama, and S. Tarucha, Nature Physics 4, 776 (2008).

[38] D. V. Khomitsky, L. V. Gulyaev, and E. Ya. Sherman, Phys. Rev. B 85, 125312 (2012).

[39] M. P. Nowak, B. Szafran, and F. M. Peeters, Phys. Rev. B 86, 125428 (2012).

[40] G. Dresselhaus, Phys.Rev. 100,580 (1955).

[41] F.G. Pikus and G.E. Pikus, Phys. Rev. B 51, 16928 (1995).

[42] Chi-Shung Tang, Shu-Yu Chang, and Shun-Jen Cheng, Phys. Rev. B 86125321 (2012).

[43] J.H. Davies, I.A. Larkin, and E.V. Sukhorukov, J. Appl. Phys. 77, 4504 (1995).

[44] D.Bjorken and D.Drell, Relativistic Quantum Mechanics (McGraw-Hill, New York, 1978), Vol.1.

[45] R. Winkler Spin-Orbit Coupling Effects in TwoDimensional Electron and Hole Systems, Springer Tracts in Modern Physics, Vol. 191 Springer-Verlag Berlin Heidelberg (2003).

[46] A.V. Moroz and C.H.W. Barnes, Phys. Rev. B 60, 14272 (1999)

[47] C.-H. Lin, C.-S. Tang, and Y.-C. Chang, Phys. Rev. B 78245312 (2008). 
[48] E.A. de Andrada e Silva and G.C. La Rocca, Phys. Rev. B 67, 165318 (2003).

[49] E.N. Bulgakov and A.F. Sadreev, Phys. Rev. B 66, 075331 (2002).

[50] F. Zhai and H. Q. Xu, Phys. Rev. Lett. 94, 246601 (2005).

[51] M. Büttiker and R. Landauer, Phys. Rev. Lett. 49, 1739 (1982).

[52] A.-P. Jauho, Phys. Rev. B 41, 12327 (1990).

[53] M. Wagner, Phys. Rev. B 57, 11899 (1998).

[54] E.N. Bulgakov and A.F. Sadreev, J. Phys.: Cond. Mat. 8, 8869 (1996).

[55] W. Li and L.E. Reichl, Phys. Rev. B 60, 15732 (1999).
[56] S. Kohler, J. Lehmann, and P. Hänggi, Phys. Rep. 406, 379 (2005).

[57] H. Sambe, Phys. Rev. A 7, 2203 (1973).

[58] U. Peskin and N. Moiseyev, J. Chem. Phys. 99, 4590 (1993).

[59] A.F. Sadreev, Phys. Rev. E 86, 056211 (2012).

[60] P.K. Tien and J.P. Gordon, Phys. Rev. 129, 647 (1963).

[61] C. P. del Valle, R. Lefebvre, and O. Atabek, Phys. Rev. A 59, 3701 ( 1999).

[62] R. Lefebvre and N. Moiseyev, Phys. Rev. A 69, 062105 (2004). 